\title{
Ac electrokinetics: a survey of sub-micrometre particle dynamics
}

\author{
N G Green $\dagger$, A Ramos $\nmid$ and H Morgan $\ddagger$ \\ $\dagger$ Departemento de Electronica y Electromagnetismo, Facultad de Fisica, Universidad de \\ Sevilla, Avenida de Reina Mercedes s/n, 41012 Sevilla, Spain \\ \$Bioelectronics Research Centre, Department of Electronics and Electrical Engineering, \\ University of Glasgow, Rankine Building, Oakfield Avenue, Glasgow G12 8LT, Scotland, UK \\ E-mail: ngreen@cica.es
}

Received 10 December 1999, in final form 28 January 2000

\begin{abstract}
Particles suspended in fluid exhibit motion when subjected to ac electric fields. The applied field results in forces on both the particles and the fluid, the study of which is referred to as ac electrokinetics. The ac electrokinetic techniques can be used for the controlled manipulation and characterization of particles, and the separation of mixtures. For sub-micrometre particles, Brownian motion is important and strong electric fields are required to overcome these effects. Planar micro-electrode arrays, fabricated using semiconductor manufacturing processes, can generate electric fields of the required strength from low potentials over a wide range of frequencies. This paper reviews and discusses sub-micrometre particle dynamics under the influence of dielectrophoretic and electrohydrodynamic forces. New experimental observations of the movement of sub-micrometre particles are also presented.
\end{abstract}

(Some figures in this article appear in colour in the electronic version; see www. iop.org)

\section{Introduction}

The term ac electrokinetics refers to the movement of particles using ac electric fields. There are a number of phenomena and forces that arise from the interaction of the field with a suspension of particles. The electric field acts through the Coulomb force on the charge of the particle to produce electrophoresis at low frequencies [1-3]. An ac field also induces a frequency-dependent dipole on a polarizable particle. The interaction of this dipole and a non-uniform field can give rise to dielectrophoresis [4-7], electrorotation $[8,9]$ and travelling-wave dielectrophoresis [10]. These dipole forces can be used to measure the internal and surface, electrical and dielectric properties of particles [5,11-13]. Electrokinetic techniques have also been used for the controlled manipulation of particles [14-16] and separation of mixtures [17-19].

Recently, ac electrokinetic methods have been applied to sub-micrometre particles. Unlike larger particles such as cells and bacteria, sub-micrometre particles are strongly influenced by thermal effects such as Brownian motion $[2,20]$. Consequently, greater forces and therefore stronger electric fields are required to move them. Microelectronic fabrication methods have been used to manufacture microelectrode arrays able to produce strong electric fields from low potentials over a wide range of frequencies. These have been successfully used for the dielectrophoretic manipulation of a variety of sub-micrometre particles, for example viruses, DNA and latex spheres [21-28]. The dielectric properties of sub-micrometre particles have been measured and characterized [29-31]. Separation of submicrometre particle mixtures has also been achieved [32-34], indicating the potential for practical applications for particles on the sub-micrometre scale.

The use of high electric field strengths produces fluid flow and heating of the suspending electrolyte $[24,35]$. The electric field can interact with the fluid to produce frequency dependent forces: electro-osmosis and electrothermal [35-37]. The resulting fluid flow exerts a drag force on the particle and therefore produces an observed motion. Recently, a new type of fluid flow has been observed on microelectrodes due to the action of the non-uniform field on the electrical double layer $[35,38-40]$. Termed ac electroosmosis, this fluid flow has been used in conjunction with dielectrophoresis for the separation of a mixture of submicrometre particles [33].

This paper briefly reviews ac electrokinetic techniques as they apply to sub-micrometre particles, together with new experimental observations of particle movement. Fluid flow patterns are reported and characterized with respect to the ac electro-osmotic and electrothermal mechanisms.

\section{Background and theory}

The study of the ac electrokinetic dynamics of particles suspended in an electrolyte requires an understanding of the interaction between the medium and the particle. The effects of the drag force, Brownian motion and particle-particle interactions are greater for colloidal particles than for larger objects. 


\subsection{Colloidal dynamics: viscous drag, Brownian motion and particle-particle interactions}

A particle moving in a fluid experiences a drag force $-f \boldsymbol{v}$ where $v$ is the velocity of the particle and $f$ is the friction factor of the particle [41]. The friction factor is related to the size, shape and surface characteristics of the particle, and for a smooth sphere is given by $f=6 \pi \eta a$ where $a$ is the radius and $\eta$ is the viscosity of the medium. Following the application of a constant force $\boldsymbol{F}$ at time $t=0$, the solution for the time dependence of the velocity is:

$$
\boldsymbol{v}=\frac{\boldsymbol{F}}{f}\left(1-e^{-(f / m) t}\right)
$$

where $m$ is the mass of the particle. The velocity of the particle increases until it reaches a constant terminal velocity. The characteristic time $\tau_{a}=m / f$ describes the acceleration phase of the particle motion (for cells and sub-micrometre particles $\tau_{a}<10^{-6} \mathrm{~s}$ ). If $\tau_{a}$ is much smaller than the typical time of observation, $\tau_{o}$, the acceleration phase is not observed and can be neglected. For observation by eye $\tau_{o}$ is $\sim 1 / 30 \mathrm{~s}$ and a sub-micrometre particle can be considered to move at the terminal velocity:

$$
\boldsymbol{v}_{t}=\boldsymbol{F} / f
$$

Therefore, any change in measured velocity is simply proportional to a change in the force.

The main external influence on a particle in a fluid is gravity, which manifests itself as the buoyancy force. For a particle of mass density $\rho_{p}$ in a fluid of density $\rho_{f}$ the buoyancy force is given by:

$$
\boldsymbol{F}_{\text {buoy }}=v\left(\rho_{p}-\rho_{f}\right) \boldsymbol{g}
$$

where $g$ is the acceleration due to gravity and $v$ is the volume of the particle. For sub-micrometre particles, this force is usually negligible but can be seen over long periods of observation (sedimentation).

Thermal effects also influence a sub-micrometre particle $[35,42]$. Since the force and velocity associated with Brownian motion have zero average, this effect can be neglected under the condition that the magnitude of the applied force $\boldsymbol{F}$ is above a certain observational threshold [42]. It can be shown that the threshold force required for 99.7\% certainty that the observed movement is due to the applied force rather than Brownian motion is

$$
|\boldsymbol{F}|=\frac{\sqrt{18 k T f}}{\sqrt{\Delta t}}
$$

where $k$ is Boltzmann's constant, $T$ is the temperature and $\Delta t$ is the period of observation. This clearly demonstrates that as the period of observation is increased, the force required to observe deterministic movement decreases. This definition and the consideration of Brownian motion in this manner are only valid for single isolated particles. If a collection of particles is being examined, diffusion of the ensemble must be considered. The driving mechanism for diffusion can be the cumulative effect of Brownian motion and particle-particle interactions $[2,43]$. The diffusion flux density of particles is

$$
j_{d}=-D \nabla n
$$

Ac electrokinetics: a survey of sub-micrometre particle dynamics

where $n$ is the concentration and $D$ is a diffusion constant including a component from Brownian motion $\left(D_{t h}=k T / f\right)$ and a contribution from particle-particle interactions. As the concentration tends to zero, $D \rightarrow D_{t h}$ and the system consists of isolated particles undergoing Brownian motion.

\subsection{Electric field in an electrolyte}

In an electrolytic solution an electrical double layer forms on the electrode surfaces $[36,43]$. When an ac signal is applied to the electrodes some of the potential is dropped across the double layer, an effect that is referred to as electrode polarization [44]. The effect is frequency dependent, with most of the applied voltage dropped across the double layer at low frequencies. Electrode polarization is negligible at high frequencies.

The potential across the medium, and the magnitude of the electric field experienced by the particle is then also frequency dependent and the observed electrokinetic forces on the particles will also be affected. This has been discussed in the ac electrokinetic literature and used to correct the measured force on particles at low frequencies [45].

\subsection{Electrical forces on the particle}

For applied potentials of a given frequency $\omega$, a suitable method of representing quantities is using phasors. An arbitrary, harmonic potential is defined as $\phi(x, t)=$ $\operatorname{Re}\left[\tilde{\phi}(\boldsymbol{x}) \mathrm{e}^{\mathrm{i} \omega t}\right]$ where $\mathrm{i}=\sqrt{-1}, \boldsymbol{x}$ is the position, $\operatorname{Re}[\ldots]$ indicates the real part of, and the tilde indicates a complex number, in this case the phasor $\tilde{\phi}=\phi_{R}+\mathrm{i} \phi_{I}$. The electric field is then given by $\boldsymbol{E}(\boldsymbol{x}, t)=\operatorname{Re}\left[\tilde{\boldsymbol{E}}(\boldsymbol{x}) \mathrm{e}^{\mathrm{i} \omega t}\right]$ where the vector $\tilde{\boldsymbol{E}}=-\nabla \tilde{\phi}=-\left(\nabla \phi_{R}+\mathrm{i} \nabla \phi_{I}\right)$ is the corresponding phasor $[46,47]$.

The dielectrophoretic force arises from the interaction of a non-uniform electric field and the dipole moment induced in the particle. For linear, isotropic dielectrics and an applied potential of a single frequency, the relationship between the electric field phasor $\tilde{\boldsymbol{E}}$ and the dipole moment phasor $\tilde{\boldsymbol{m}}(\omega)$ for a spherical particle is [6]:

$$
\tilde{\boldsymbol{m}}(\omega)=v \tilde{\alpha}(\omega) \tilde{\boldsymbol{E}}
$$

where $\tilde{\alpha}$ is referred to as the effective polarizability of the particle [5] and $v$ is the volume of the particle. The timeaveraged force on the particle is given by [6]

$$
\left\langle\boldsymbol{F}_{d e p}\right\rangle=\frac{1}{2} \operatorname{Re}\left[(\tilde{\boldsymbol{m}} \cdot \nabla) \tilde{\boldsymbol{E}}^{*}\right]
$$

where * indicates complex conjugate. Using vector identities and the facts that the applied electric field is irrotational and has zero divergence, the force can be expressed as:

$$
\begin{gathered}
\left\langle\boldsymbol{F}_{\text {dep }}\right\rangle=\frac{1}{4} v \operatorname{Re}[\tilde{\alpha}] \nabla|\tilde{\boldsymbol{E}}|^{2}-\frac{1}{2} v \operatorname{Im}[\tilde{\alpha}] \\
\times(\nabla \times(\operatorname{Re}[\tilde{\boldsymbol{E}}] \times \operatorname{Im}[\tilde{\boldsymbol{E}}]))
\end{gathered}
$$

where $\operatorname{Im}[\ldots]$ is the imaginary part of, and $|\tilde{\boldsymbol{E}}|^{2}=\tilde{\boldsymbol{E}} \cdot \tilde{\boldsymbol{E}}^{*}$. The first term on the right-hand side is non-zero if there is a spatially varying field magnitude, giving rise to dielectrophoresis $[4,5]$. The second term is non-zero if 
there is a spatially varying phase, as in the case of travelling wave dielectrophoresis $[10,48]$. The second term should also be considered if the electric field is affected by electrode polarization, since the phasor for the potential across the medium has non-zero real and imaginary components.

For dielectrophoresis alone, the velocity of a submicrometre particle, using equation (2), is given by [34]:

$$
\boldsymbol{v}_{d e p}=\mu_{d e p} \nabla|\tilde{\boldsymbol{E}}|^{2}=\frac{v \operatorname{Re}[\tilde{\alpha}]}{4 f} \nabla|\tilde{\boldsymbol{E}}|^{2}
$$

where the dielectrophoretic mobility $\mu_{\text {dep }}$ has the units $m^{4} \mathrm{~s}^{-1} \mathrm{~V}^{-2}$. Considering only Maxwell-Wagner interfacial polarization, the dielectrophoretic mobility of a spherical particle of radius $a$ is:

$$
\mu_{d e p}=\frac{a^{2} \varepsilon_{m}}{6 \eta} \operatorname{Re}\left[\frac{\tilde{\varepsilon}_{p}-\tilde{\varepsilon}_{m}}{\tilde{\varepsilon}_{p}+2 \tilde{\varepsilon}_{m}}\right]
$$

where the subscripts $m$ and $p$ indicate medium and particle respectively. $\tilde{\varepsilon}$ indicates a complex permittivity: $\tilde{\varepsilon}=\varepsilon-$ $\mathrm{i} \sigma / \omega$ where $\varepsilon$ is the permittivity, $\sigma$ is the conductivity and $\omega$ is the frequency. The part of the expression in brackets is referred to as the Clausius-Mossotti factor and describes the frequency variation of the dielectrophoretic mobility and force. This factor varies between +1 and $-1 / 2$ and the particle will move towards (positive dielectrophoresis) or away from (negative dielectrophoresis) regions of high field strength depending on frequency.

\subsection{Electric forces on the fluid}

Electrical power is generated in the fluid to a degree proportional to the conductivity of the medium and the field magnitude squared. This can give rise to fluid flow in one of two ways. The electric field generates a temperature gradient in the fluid and therefore gradients in density, permittivity and conductivity. The first mechanism that produces a force on the fluid is natural convection where the denser fluid elements displace less dense. The second is electrothermal, where the electric field interacts with the gradients in permittivity and conductivity to produce electrical forces $[46,49]$.

However, as discussed by Ramos et al [35], the electrothermal driven fluid flow is not responsible for the fluid motion which is observed at low frequencies $(<0.5 \mathrm{MHz})$. At these frequencies, the high fluid flow velocities can be explained through the action of ac electro-osmosis [38-40,50]. When a dc field is applied tangential to an electrode surface, the ions in the double layer experience a force. The resulting movement of ions acts through friction with the fluid to produce a flow referred to as electro-osmosis [36]. A similar phenomenon occurring in ac fields has been described for planar microelectrode arrays $[38,50]$. The applied potential on the electrodes gives rise to an induced double layer and the tangential component of the generated electric field interacts with the induced charge close to the surface of the electrodes. The force acts always in the same direction, varying with the field frequency, field magnitude and medium conductivity.

\subsection{Summary of particle dynamics}

The dynamics of a colloidal particle in divergent electric fields are complicated and analysis of experimental observations can be difficult. For example, the electrothermal fluid flow competes with the dielectrophoretic force at high frequencies, and at low frequencies ac electro-osmotic flow dominates and this is strong even for low potentials. On top of these applied forces, particle-particle interactions and Brownian motion occur continuously.

What is normally observed and measured in electrokinetic experiments is the position of particles as a function of time. This can be analysed to give the velocity of individual particles, which should be modelled theoretically using the Langevin equation [2]. In this case, the particle experiences random Brownian motion and such particle-particle interactions that exist, in addition to the externally applied forces.

If an ensemble of particles is being observed, the velocity of individual particles is difficult to assign correctly and the flux of the ensemble across an experimental line or surface should instead be considered. The flux density of particles, $j$, is given by:

$$
\boldsymbol{j}=\frac{n}{f}\left(\boldsymbol{F}_{\text {dep }}+\boldsymbol{F}_{\text {buoy }}\right)+n \boldsymbol{v}_{f}+\boldsymbol{j}_{d}
$$

where $\boldsymbol{F}_{d e p}$ is the dielectrophoretic force (equation (7)), $\boldsymbol{F}_{\text {buoy }}$ is the buoyancy force (equation (3)), $j_{d}$ is the diffusion flux density (equation (5)) and $\boldsymbol{v}_{f}$ is the velocity of the fluid. In order to describe the change in $n$ with time (the dynamics of the system), the continuity equation:

$$
\frac{\partial n}{\partial t}+\nabla \cdot j=0
$$

should be solved.

\section{Experimental details}

Sub-micrometre ac electrokinetic particle manipulation is performed on micrometre-scale electrodes. Two designs of electrodes were used in this work, shown schematically in figure 1. The polynomial design [51] is shown in figure 1(a), and has been used for the characterization and manipulation of cells and bacteria. It has four electrodes with edges defined by a hyperbolic function in the centre and parallel edges out to an arbitrary distance. This electrode design has been demonstrated to be a versatile tool for particle observation and characterization, applied to sub-micrometre latex spheres $[29,31]$ and viruses [26,34]. The castellated design [12] (figure 1(b)) consists of square features on parallel wires and has been used for characterization and separation. This design has been used for manipulation and separation of sub-micrometre spheres [32-34]. Both designs have welldefined electric field patterns giving clearly distinct regions for positive and negative dielectrophoresis $[12,52]$.

Electrodes were fabricated using electron-beam lithography (Leica EBPG HR-5 beamwriter) and lift-off, with feature sizes and gaps down to $1 \mu \mathrm{m}$. Figure 2 shows a scanning electron micrograph of castellated electrodes fabricated by this method. The electrodes consist of layers of $10 \mathrm{~nm}$ titanium, 

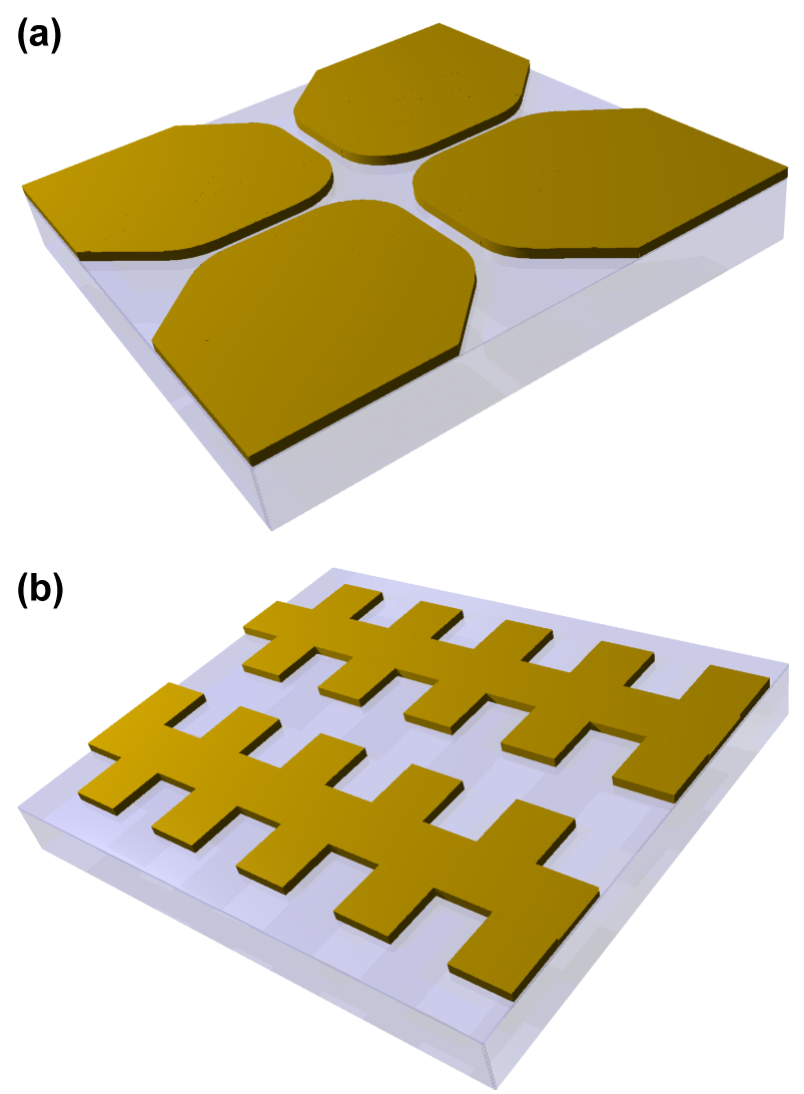

Figure 1. Schematic diagram of two designs of electrodes: (a) the polynomial design has four electrodes with edges defined by a hyperbolic function in the centre and parallel edges out to an arbitrary distance and (b) the castellated design consists of square features on parallel wires.

$10 \mathrm{~nm}$ palladium, $100 \mathrm{~nm}$ gold and $20 \mathrm{~nm}$ titanium. The upper layer of titanium allowed the electrodes to be used at low frequencies $(\sim 1 \mathrm{~Hz})$ in conductive ionic media. The edges of the electrodes were well defined, with feature resolutions of approximately $25 \mathrm{~nm}$ giving sharp corners to the castellations.

The particles used in the experiments were fluorescently loaded latex spheres purchased from Molecular Probes (Eugene, Oregon, USA) suspended in electrolytic solutions. The diameters of the spheres were in the range $557 \mathrm{~nm}$ to $93 \mathrm{~nm}$. The potential signals were generated using both analogue and digital signal generators. The particle response was observed using a fluorescence microscope (Nikon Microphot) and a digital camera/video system.

\section{Electric field simulation}

The electrokinetic response of the particles depends on the strength and spatial variation of the electric field. In order to compare theory and experiment, the electric field must be known in three dimensions. The electric field in the electrode arrays was numerically simulated using the commercial finite element solver, Maxwell (Ansoft, UK). Figure 3 shows examples of the field patterns calculated for the electrode designs shown in figure 1 from [52]. The polynomial electrodes (figure 3(a)) have a local field

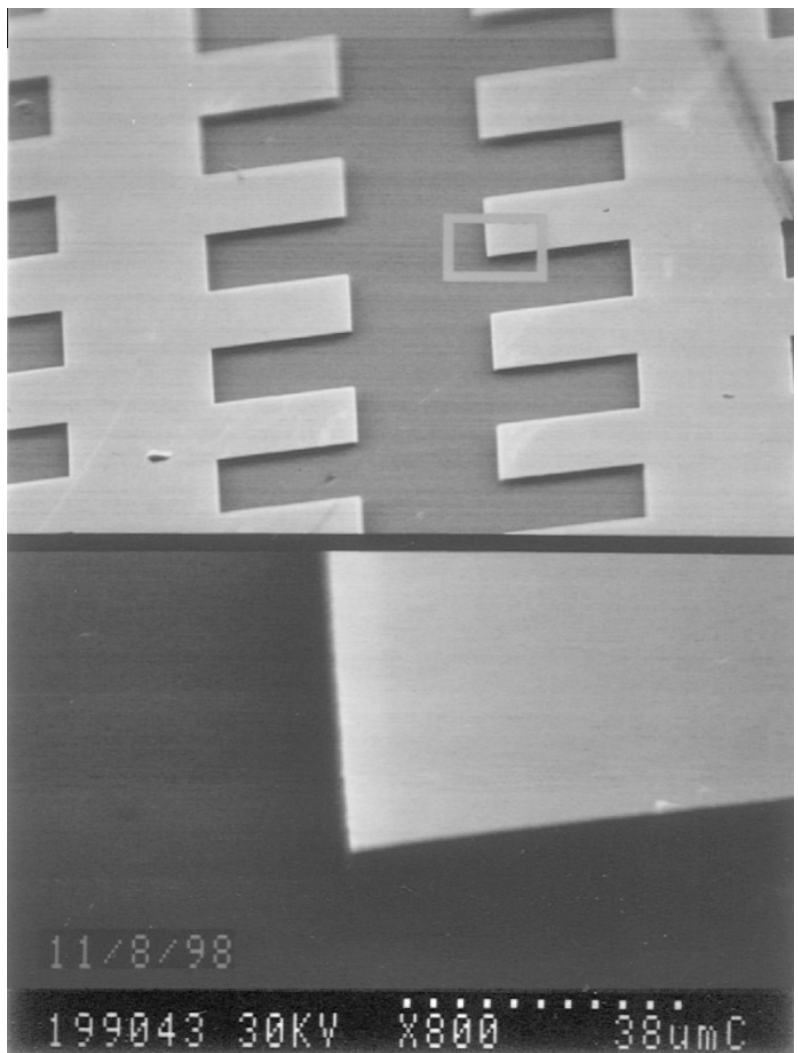

Figure 2. A scanning electron microscope image of a set of castellated electrodes manufactured using direct write e-beam lithography.

minimum and negative dielectrophoretic trap in the centre of the four electrodes (marked as point A) and high field points along the edges of the electrodes (marked B), especially where adjacent electrodes run parallel. The castellated electrodes (figure 3(b)) have local field minima in the bays between the castellations (marked A) which form negative dielectrophoretic traps. There are high field regions in the gap between electrodes, particularly along the front edges of the castellations (marked B) and also a smaller high field region at the back of the bays (marked C). Particles experiencing positive dielectrophoresis collect at high field points across the electrode array. These results are consistent with previous simulations using other techniques $[12,51,53]$.

A point of particular interest for sub-micrometre particles, where the force due to gravity is comparatively small, is that the local field minimum in each bay of the castellated electrode is a closed 3D minimum. This is illustrated by figure 3(c) which shows the electric field magnitude in a vertical plane through the line marked between points (i) and (ii) in figure 3(b). The minimum (point A) extends approximately half the size of the castellated features away from the electrodes. For particles experiencing negative dielectrophoresis this field minimum forms a 3D trap or 'cage'. Above the trap the particles experience an almost uniform negative dielectrophoresis (DEP) force pushing them away from the electrodes. In this electrode design, multiple closed, 3D negative DEP traps can be generated over a large area without the need for complicated three-dimensional electrode structures [53]. 


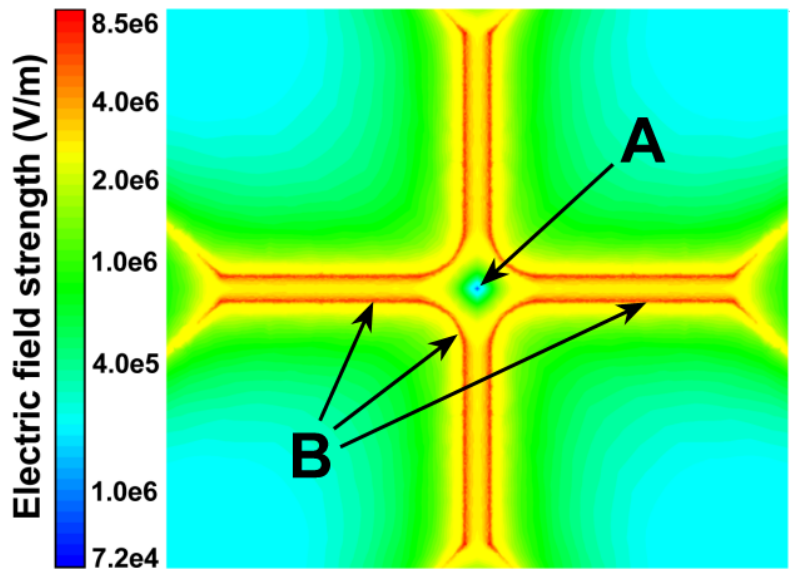

(a)

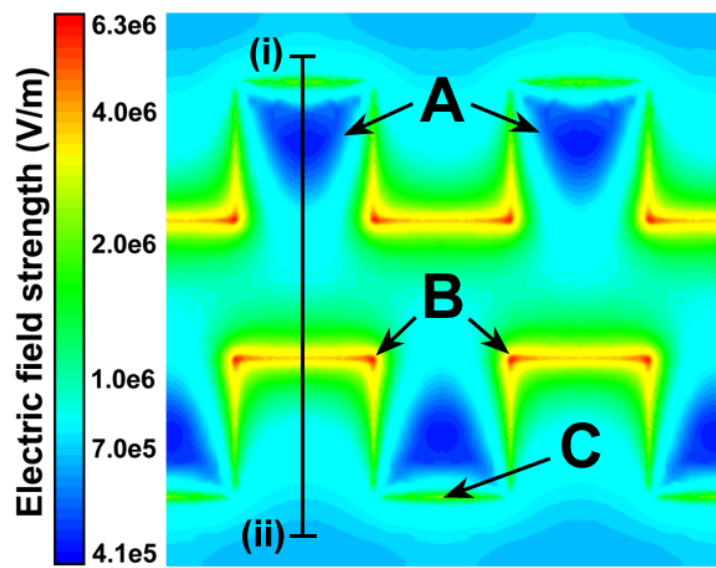

(b)

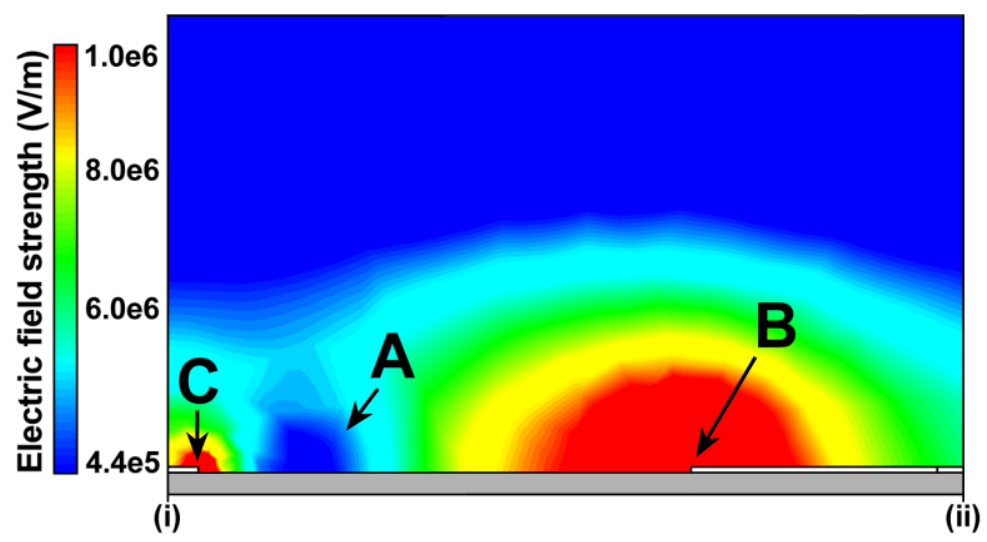

(c)

Figure 3. (a) Plot of the numerically calculated electric field in a horizontal plane $100 \mathrm{~nm}$ above polynomial electrodes. The distance between the parallel edges of the electrodes was $2 \mu \mathrm{m}$ and the distance across the centre of the four (across point A) was $6 \mu \mathrm{m}$. The applied potential was \pm 5 volts applied to adjacent electrodes. There is a local low field minimum in the centre (point A) which is a negative dielectrophoretic trap. There are high field regions along each electrode edge (points B) which will collect particles experiencing positive dielectrophoresis. (b) Plot of the numerically calculated electric field in a horizontal plane $100 \mathrm{~nm}$ above castellated electrodes. The distance between the electrodes and the size of the square feature was $5 \mu \mathrm{m}$ and the applied potential was \pm 5 volts on either electrode. There are local 3D field minima in each bay between castellations (points A) which are negative dielectrophoretic traps. There are high field regions in the gap between electrodes, particularly along the front edges of the castellations (points B) and also a smaller high field region at the back of the bays (points $\mathrm{C}$ ). (c) Plot of the numerically calculated electric field in a vertical plane along the line between the points marked (i) and (ii) in figure 3(b). The trap (point A) extends approximately half the size of the castellated features away from the electrodes. Above this height particles experience an almost uniform negative DEP force pushing them away from the electrodes.

\section{Results and discussion}

\subsection{Dielectrophoresis}

Figures 4(a) and (b) show photographs of negative dielectrophoresis of $557 \mathrm{~nm}$ diameter latex spheres on polynomial and castellated electrodes. As indicated by the field simulation for the polynomial electrodes (figure 4(a)), the spheres collected in the centre of the electrodes and were repelled from the electrode edges $(5 \mathrm{MHz}, 5$ volts peakto-peak). Figure 4(b) shows triangular collections of latex spheres on castellated electrodes as predicted by the electric field model at a frequency of $8 \mathrm{MHz}$ and 8 volts peak-to-peak.

Figures 4(c) and (d) show positive dielectrophoresis of $557 \mathrm{~nm}$ diameter latex spheres on the same electrode designs. In the polynomial electrodes at $500 \mathrm{kHz}$ and 5 volts, the particles collected along the electrode edges (figure 4(c)). Figure 4(d) shows positive dielectrophoresis on the castellated electrodes with the particles collecting at the front edge of the electrodes (point B in figure 3(b)). However, as the particles were previously held by negative DEP, as shown in figure 4(b), once the frequency was changed to allow positive DEP, the particles moved to the closest high field point. In this case, the particles collected at the back of the bay area at point C (figure 3(b)), consistent with the field plot.

\subsection{Fluid flow}

During the observation of particle movement in high electric fields, a pattern of behaviour is seen that does not conform to that expected from dielectrophoresis. This motion is believed to be due to fluid flow, since it is similar over a range of particle sizes and the observations of circular patterns point to some form of convection.

5.2.1. High frequencies At high frequencies (above $100 \mathrm{kHz}-1 \mathrm{MHz}$, depending on the medium conductivity) 


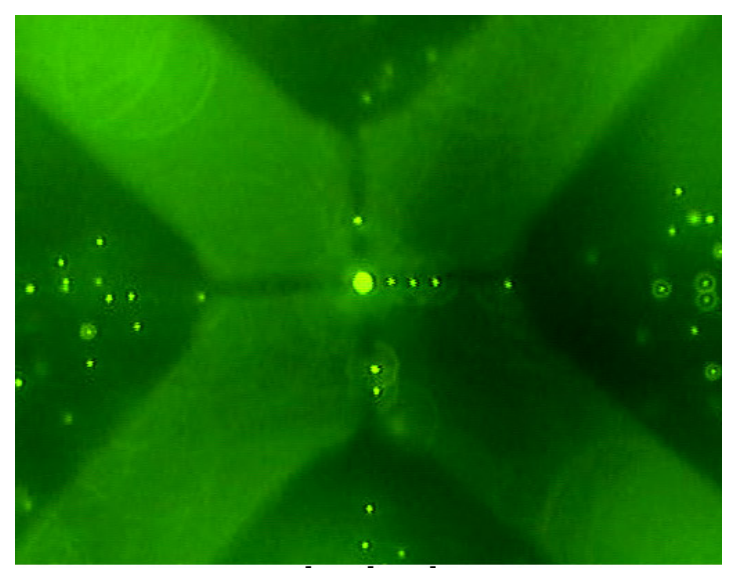

$20 \mu \mathrm{m}$

(a)

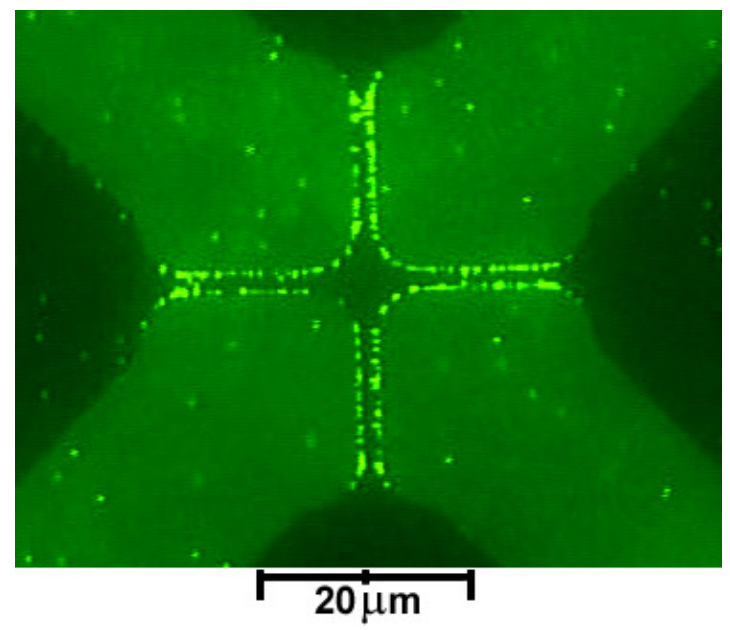

(c)

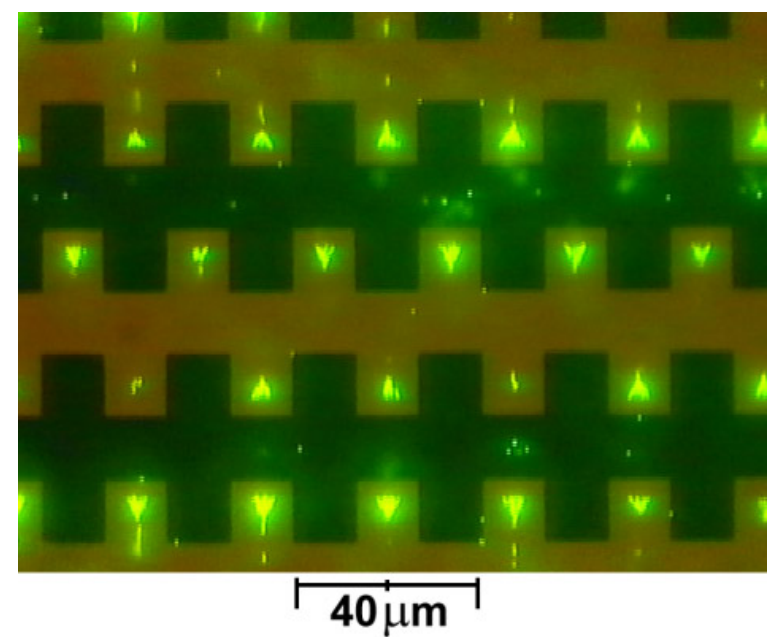

(b)

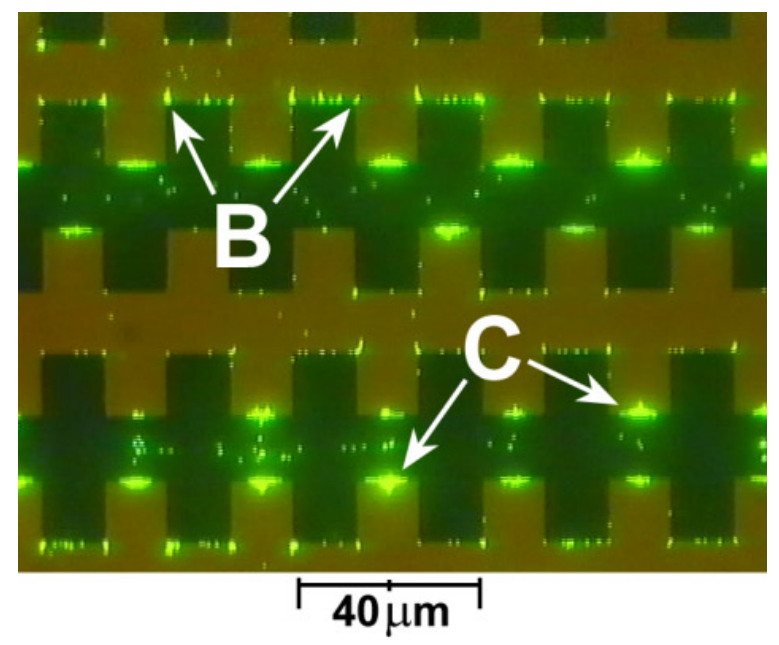

(d)

Figure 4. (a) Negative dielectrophoresis of $557 \mathrm{~nm}$ diameter latex spheres on polynomial electrodes for an applied signal of 5 volts peak-to-peak at $5 \mathrm{MHz}$. The spheres can clearly be seen collecting in the low field region as indicated by point A in figure 3(a). (b) Negative dielectrophoresis of $557 \mathrm{~nm}$ diameter latex spheres on castellated electrodes for an applied signal of 8 volts peak-to-peak at $8 \mathrm{MHz}$. The spheres can clearly be seen collecting in the low field regions indicated by points A in figure 3(b). (c) Positive dielectrophoresis of 557 nm diameter latex spheres on polynomial electrodes with an applied signal of 5 volts peak-to-peak at $500 \mathrm{kHz}$. The spheres collect along the edges of the electrodes at the high field points indicated by points B in figure 3(a). (d) Positive dielectrophoresis of $557 \mathrm{~nm}$ diameter latex spheres on castellated electrodes with an applied signal of 8 volts peak-to-peak at $700 \mathrm{kHz}$. The spheres collect at the high field regions marked as points B in figure 3(b) and also at the high field points at the back of the bays (points C).

distinct patterns of fluid flow were observed. For the polynomial electrodes, fluid was observed to move into the centre of the electrode array, as illustrated in figure 5(a). This effect was observed for frequencies above $10 \mathrm{MHz}$ for the experimental conductivities used (up to $\sim 100 \mathrm{mS} \mathrm{m}^{-1}$ ) and is difficult to distinguish from negative dielectrophoresis. Similar flow patterns were observed by Müller et al [24] who attributed the effect to the electrothermal mechanism. In this work however, the direction and pattern of the flow was observed to vary with both the frequency and potential of the applied field. In figure 5(b) the pattern of fluid behaviour at lower frequencies (around 3-4 MHz) and low potentials (5 volts peak-to-peak) is illustrated. Particles are still observed to move towards the centre of the array from the bulk but also down into the centre of the electrode array and outwards. This results in four regions where particles collect over the gaps between the electrodes, as illustrated. When either the potential or the frequency (or both) was increased, the velocity of the particles moving into the electrode array increased and the four collections of particles moved into the centre of the electrode array, as in figure 5(a). When the signal was changed back to the original settings, the particles returned to collecting at the four points.

Figure 5(c) shows an experimental image of this flow for $282 \mathrm{~nm}$ diameter spheres with a potential of 12 volts peak-to-peak and $6 \mathrm{MHz}$. The particles at the four collection points whirl in circular patterns in a plane perpendicular to the electrode surface and the gap. Figure 5(d) shows the flow pattern for $557 \mathrm{~nm}$ diameter latex spheres, for a potential of 5 volts peak-to-peak and a frequency of $3 \mathrm{MHz}$. Some particles are trapped in the centre of the electrodes by negative dielectrophoresis (point A) and some are pushed to the four collection points (points B). Outside the electrodes, the drag force due to fluid flowing into the electrode arrays is balanced 


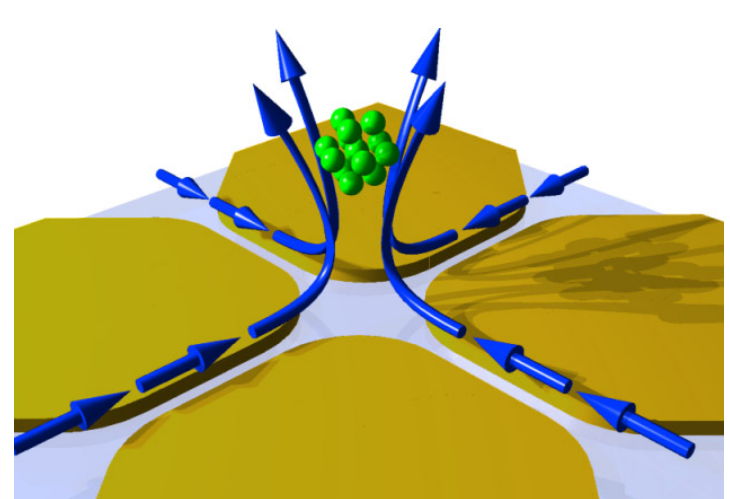

(a)

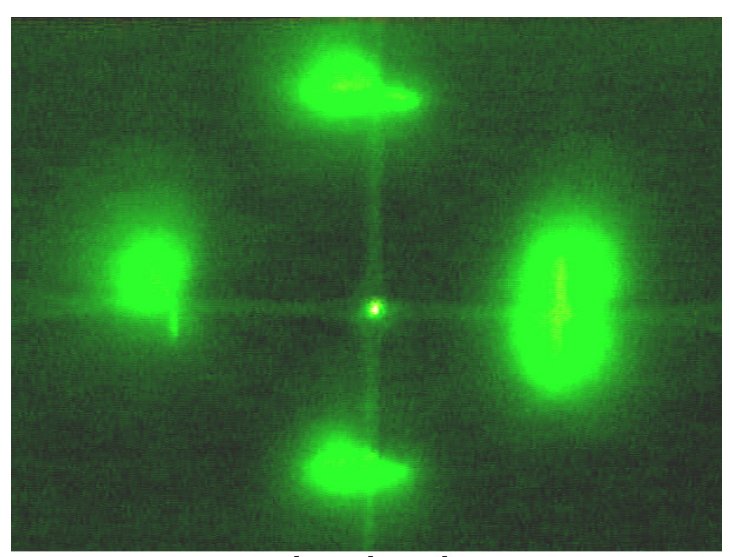

$20 \mu \mathrm{m}$

(c)

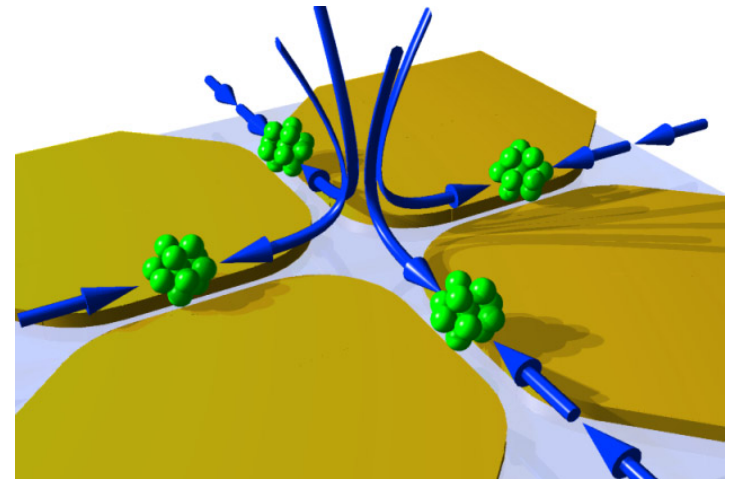

(b)

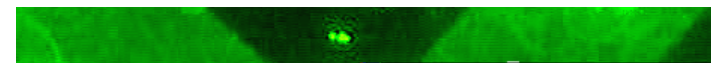

(d)

Figure 5. (a) A schematic diagram of the fluid flow observed in polynomial electrode arrays at high frequencies. The fluid moves into the electrode array along the gaps between adjacent electrodes and then up and out in the centre. The particles are typically pushed along with the fluid flow and settle in a levitated position above the centre of the array. If this fluid flow was stronger than the dielectrophoretic force, this collection in the centre is observed if the particles are experiencing either positive or negative dielectrophoresis. (b) A schematic diagram of the pattern of fluid behaviour at lowers frequencies (around 3-4 MHz) and low potentials (5 volts peak-to-peak). There is flow towards the centre of the array from the bulk but also a flow down into the centre of the electrode array and outwards. This results in four regions where particles collect over the gaps between the electrodes. When either the potential or the frequency (or both) was increased, the flow velocity into the electrode array increased and the four collections of particles moved into the centre of the electrode array, as in figure 5(a). (c) An experimental image of the flow described by figure 5(b) for $282 \mathrm{~nm}$ diameter spheres at a potential of 12 volts peak-to-peak and $6 \mathrm{MHz}$. The particles at the four collection points whirl in circular patterns in a plane perpendicular to the electrode surface and the gap. (d) An experimental image of the flow pattern for $557 \mathrm{~nm}$ diameter latex spheres, for a potential of 5 volts peak-to-peak and a frequency of 3 $\mathrm{MHz}$. Some particles are trapped in the centre of the electrodes by negative dielectrophoresis (point A) and some are pushed to the four fluid collection points by fluid flow (points B). Outside the electrodes, the negative DEP force pushing the particles away from the electrodes balances the drag force due to the fluid flowing into the electrode arrays (points $\mathrm{C}$ ).

by the negative DEP force pushing the particles away from the electrodes (points C). If either the frequency or potential was increased, the pattern of particle movement changed to that shown in figure 5(a).

Although the fluid flow observed at high frequencies is thought to be electrothermal, in general the electrical power dissipated in the medium (Joule heating) cannot produce a sufficiently large temperature gradient [35]. However, the magnitude of some other types of fluid flow at these frequencies has been found to depend on the intensity of the illumination. When the illumination intensity was reduced, the flow magnitude decreased, indicating that the light produces temperature gradients around the electrodes. This is discussed in more detail in a related publication [54]. It should be noted at this point that the collection of particles in the four points in the polynomial electrodes was found to occur independently of the illumination intensity.

In addition to these large-scale fluid movements, smallscale movement of fluid was observed close to electrode edges. The flow patterns consisted of cylindrical rolls of the order of 1-5 $\mu \mathrm{m}$ in diameter over electrode edges (at the point of maximum field). These patterns were observed for field strengths over $10^{6} \mathrm{Vm}^{-1}$ and the magnitude of the effect increased rapidly with increasing applied potential. The direction of the rolls changed at a particular frequency of the applied field. This latter observation strongly suggests that this is related in some way to the electrothermal effect, since theory predicts [35] a change in direction of fluid flow at a given frequency. Also, the low order of magnitude of the velocity of this flow agrees with the predicted order of magnitude of electrothermal flow powered by Joule heating alone [35]. 

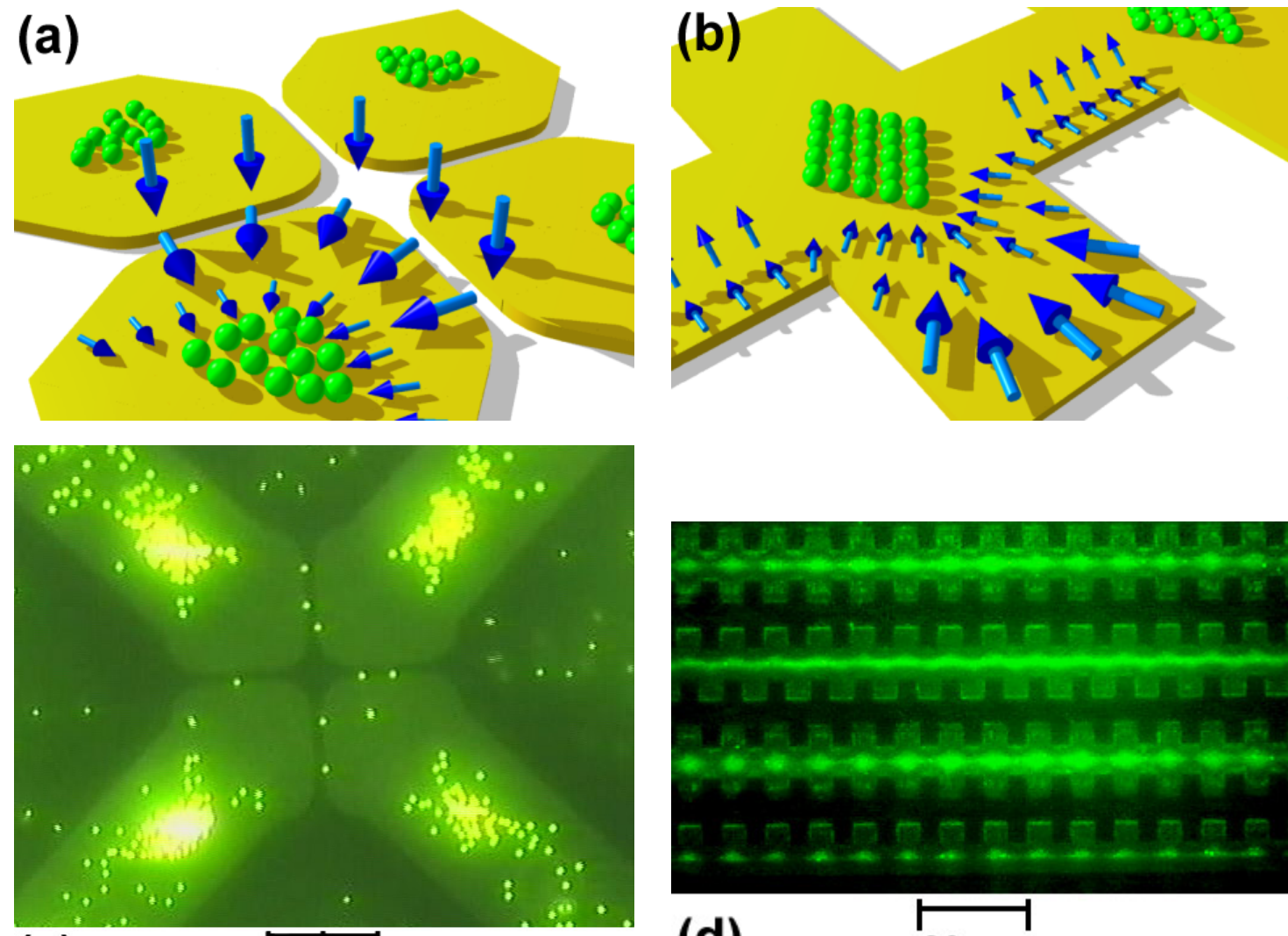

(c)
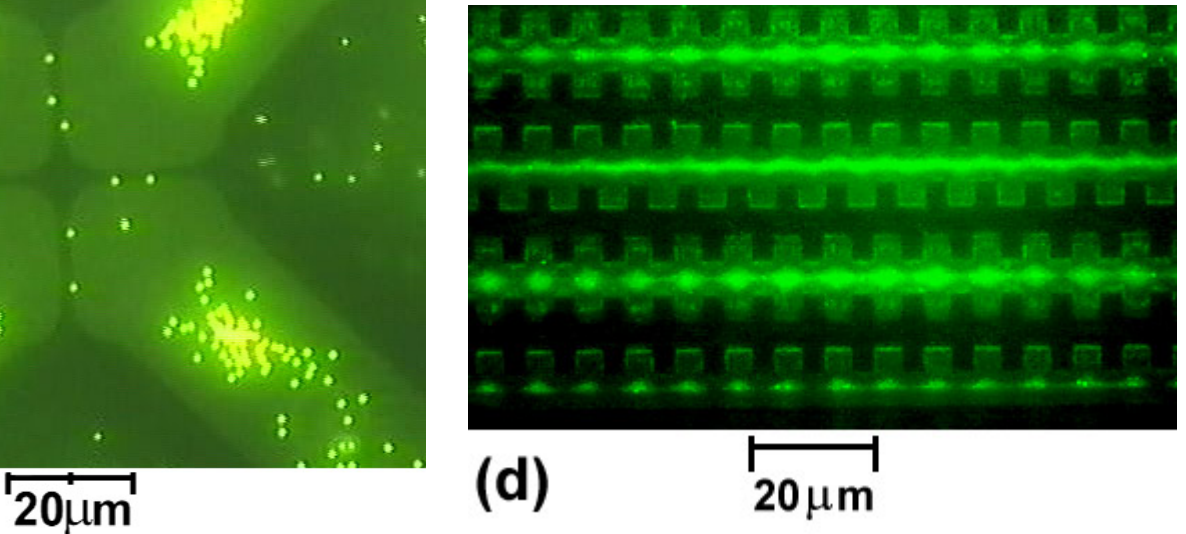

Figure 6. Diagrams and experimental images of ac electro-osmosis for two designs of electrodes: (a), (b) schematic diagrams of the fluid flow patterns on the polynomial and castellated electrode respectively; (c) on the polynomial electrodes, the particles are pushed away from the edges, collecting on top of the electrodes; (d) on the castellated electrodes, the fluid pushes the particles into diamond shaped collections along the symmetry line of the electrodes.

\subsubsection{Low frequencies Figures 6(a) and (b) show} schematic diagrams of ac electro-osmosis for the two designs of electrodes and figures 6(c) and (d) show experimental images of the effect of the fluid flow on particles. At frequencies of the order of $1-10 \mathrm{kHz}$ on the polynomial electrodes, the particles are pushed away from the edges, collecting on top of the electrodes. On the castellated electrodes, the fluid pushes the particles into diamond shaped collections along the symmetry line of the electrodes, an effect which has been reported previously [12], but which was attributed to anomalous dielectrophoretic effects.

This type of fluid flow depends to a great extent on the frequency of the applied field, typically rising and then falling in magnitude as the frequency is decreased [38,39]. The value of the frequency at which the velocity has maximum magnitude also increases with the conductivity of the fluid. In the range of frequencies where the flow is strongest and at the potentials used in these experiments, the force exerted by the fluid on the particles was more than sufficient to override the dielectrophoretic force. The experimental images showing these effects for the polynomial electrode and the castellated electrodes are presented in figures 6(c) and (d). The experimental conditions were a frequency of $100 \mathrm{kHz}$, an applied potential of 5 volts peak-to-peak and a medium conductivity of $5 \mathrm{mS} \mathrm{m}^{-1}$.

\subsection{Summary of results}

All the forces illustrated in this paper occur under the same experimental conditions. However, since the magnitudes of the forces are frequency dependent, even a slight change in frequency can have a marked effect on the behaviour of the particles. As an illustration, figure 7 shows the change in behaviour of $557 \mathrm{~nm}$ diameter spheres over three decades of frequency on castellated electrodes at an applied potential of 8 volts peak-to-peak and a solution conductivity of $2 \mathrm{mS} \mathrm{m}{ }^{-1}$. Under these experimental conditions, only dielectrophoresis and ac electro-osmosis were observed. At $8 \mathrm{MHz}$ (figure 7(a)), particles experienced negative DEP and collected in triangular shapes in every bay. In figure 7(b), after switching to $0.7 \mathrm{MHz}$, the particles collected at high field points on the electrode edges under conditions of positive DEP. The diamonds and wavy stripes in figure 7 (c) at $70 \mathrm{kHz}$ are caused by the combination of positive DEP and an ac electro-osmotic flow. Where the DEP force is strongest (at the tips of the castellations) particles remain trapped. For the high field points at the back of the bays, there is sufficient flow to push the particles away from the edges to a point where the DEP and drag forces balance. This produces diamond shapes on the symmetrical electrodes where the electrodes are narrow and wavy stripes on the asymmetrical electrodes. One decade lower, at $7 \mathrm{kHz}$ (figure 7(d)), the velocity of the fluid is the dominant effect and the particles 

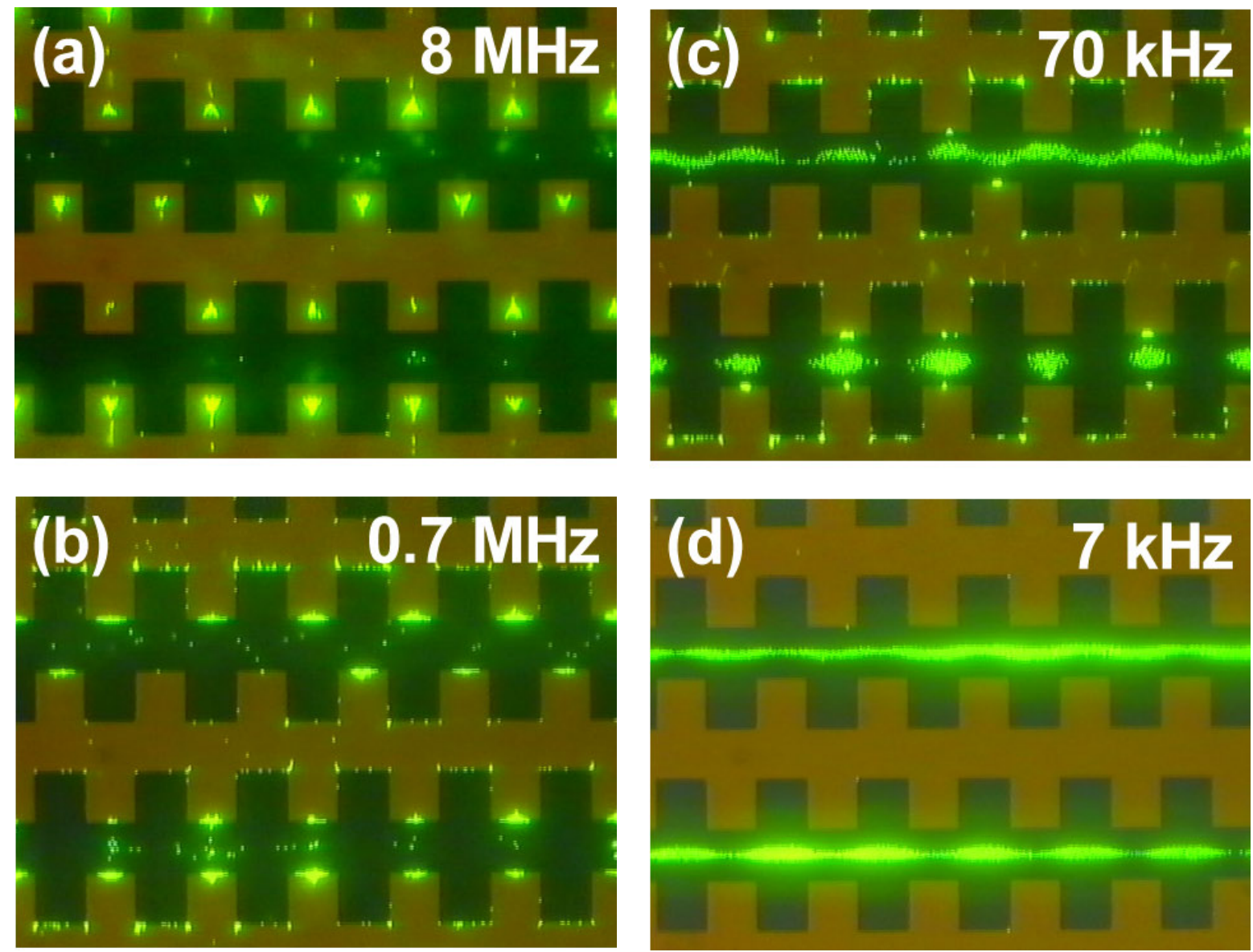

Figure 7. Experimental images of $557 \mathrm{~nm}$ diameter spheres over three decades of frequency on castellated electrodes at an applied potential of 8 volts peak-to-peak and a solution conductivity of $2 \mathrm{mS} \mathrm{m}^{-1}$. At $8 \mathrm{MHz}$, (a), particles experience negative DEP and collect in triangular shapes in every bay. At $0.7 \mathrm{MHz},(\mathrm{b})$, the particles collect at high field points on the electrode edges under conditions of positive DEP. The diamonds and wavy stripes in figure 7(c) at $70 \mathrm{kHz}$ are caused by the combination of positive DEP and an ac electro-osmotic flow. Where the DEP force is strongest (points B in figure 3(b)) particles remain trapped but at the high field points at the back of the bays (points C), there is sufficient flow to push the particles away from the edges. This produces diamond shapes on the symmetrical electrodes where the electrodes are narrow and wavy stripes on the asymmetrical electrodes. At $7 \mathrm{kHz},(\mathrm{d})$, the velocity of the fluid is the dominant effect and the particles are pushed onto the electrodes to form diamond shapes at the widest part of the symmetrical electrodes and wavy lines where the electrodes are asymmetrical.

are pushed onto the electrodes to form diamond shapes at the widest part of the symmetrical electrodes and wavy lines where the electrodes are asymmetrical. This explains the anomalous effects observed in previous dielectrophoretic experiments [12].

\section{Conclusion}

The ac electrokinetic forces that a sub-micrometre particle experiences in spatially non-uniform ac electric fields on microelectrodes have been outlined and discussed. The results of particle motion under the influence of these forces as reported in the literature have been reviewed. The major effects observed in the movement of sub-micrometre particles on microelectrodes have been discussed and illustrated with new experimental observations. Unexplained phenomena reported previously in the literature have been clarified in terms of this summary of observed effects.

The ac electrokinetic techniques have the potential for being a major technology for particle measurement and separation. The capability of these techniques for submicrometre particle characterization and/or measurement has been demonstrated [23, 24, 27, 29,31-34], particularly for viruses $[25,26,30,34]$. The electrokinetic separation of mixtures of sub-micrometre particles has been achieved using dielectrophoresis $[32,34]$ and also in combination with electrically induced fluid flow [33].

\section{Acknowledgments}

The authors would like to thank the Engineering and Physical Sciences Research Council, UK for providing a studentship and the European Union for awarding a Marie Curie fellowship (contract no. BIO4-CT98-5010 (DG12SSMI)) to N G Green. They would also like to thank Professor A Castellanos and Dr A Gonzalez for valuable discussions and Mr W Monaghan and Ms M Robertson for technical assistance. 


\section{References}

[1] Grossman P D and Colburn J C 1992 Capillary Electrophoresis (New York: Academic)

[2] Russel W B, Saville D A and Schowalter W R 1995 Colloidal Dispersions (Cambridge: Cambridge University Press)

[3] Rider P F and O’Brien R W 1993 J. Fluid Mech. 257 607-36

[4] Pohl H A 1951 J. Appl. Phys. 22869

[5] Pohl H A 1978 Dielectrophoresis (Cambridge: Cambridge University Press)

[6] Jones T B 1995 Electromechanics of Particles (New York: Cambridge University Press)

[7] Pethig R 1996 Crit. Rev. Biotech. 16 331-48

[8] Arnold W M, Schwan H P and Zimmermann U 1987 J. Phys. Chem. 91 5093-8

[9] Wang X B, Pethig R and Jones T B 1992 J. Phys. D: Appl. Phys. 25 905-12

[10] Hagedorn R, Fuhr G, Müller T and Gimsa J 1992 Electrophoresis 13 49-54

[11] Pohl H A and Crane J S 1971 Biophys. J. 11 711-27

[12] Pethig R, Huang Y, Wang X B and Burt J P H 1992 J. Phys. D: Appl. Phys. 25 881-8

[13] Wang X B, Huang Y, Gascoyne P R C and Becker F F 1997 IEEE Trans. Ind. Appl. 33 660-9

[14] Kaler K, Xie J P, Jones T B and Paul R 1992 Biophys. J. 63 58-69

[15] Washizu M, Kurahashi Y, Iochi H, Kurosawa O, Aizawa S, Kudo S, Magariyama Y and Hotani H 1993 IEEE Trans. Ind. Appl. 29 286-94

[16] Fuhr G, Glasser H, Müller T and Schnelle T 1994 Biochim. Biophys. Acta 1201 353-60

[17] Gascoyne P R C, Huang Y, Pethig R, Vykoukal J and Becker F F 1992 Meas. Sci. Technol. 3 439-45

[18] Markx G H, Talary M S and Pethig R 1994 J. Biotechnol. 32 29-37

[19] Markx G H, Dyda P A and Pethig R 1996 J. Biotechnol. 51 175-80

[20] Einstein A 1905 Ann. Phys., Lpz. 17 549-60

[21] Washizu M, Suzuki S, Kurosawa O, Nishizaka T and Shinohara T 1994 IEEE Trans. Ind. Appl. 30 835-43

[22] Washizu M, Kurosawa O, Arai I, Suzuki S and Shimamoto N 1995 IEEE Trans. Ind. Appl. 31 447-56

[23] Müller T, Gerardino A M, Schnelle T, Shirley S G, Fuhr G, Degasperis G, Leoni R and Bordoni F 1995 Nuovo Cimento Soc. Ital. Fisica D 17 425-32

[24] Müller T, Gerardino A, Schnelle T, Shirley S G, Bordoni F, DeGasperis G, Leoni R and Fuhr G 1996 J. Phys. D: Appl. Phys. 29 340-9

[25] Green N G, Morgan H and Milner J J 1997 J. Biochim. Biophys. Methods 35 89-102

[26] Hughes M P, Morgan H, Rixon F J, Burt J P H and Pethig R 1998 Biochim. Biophys. Acta 1425 119-26

[27] Hughes M P and Morgan H 1998 J. Phys. D: Appl. Phys. 31 2205-10
[28] Bakewell D, Morgan H and Milner J J 1999 Electrostatics 99 (Cambridge, UK) (Inst. Phys. Conf. Ser.) 163 73-6

[29] Green N G and Morgan H 1997 J. Phys. D: Appl. Phys. 30 2626-33

[30] Morgan H and Green N G 1997 J. Electrostatics 42 279-93

[31] Green N G and Morgan H 1999 J. Phys. Chem. B 103 41-50

[32] Green N G and Morgan H 1997 J. Phys. D: Appl. Phys. 30 L41-4

[33] Green N G and Morgan H 1998 J. Phys. D: Appl. Phys. 31 L25-30

[34] Morgan H, Hughes M P and Green N G 1999 Biophys. J. 77 516-25

[35] Ramos A, Morgan H, Green N G and Castellanos A 1998 J. Phys. D: Appl. Phys. 31 2338-53

[36] Hunter R J 1981 Zeta Potential in Colloid Science (London: Academic)

[37] Castellanos A 1998 Electrohydrodynamics (Udine: CISM)

[38] Ramos A, Morgan H, Green N G and Castellanos A 1999 J. Colloid Interface Sci. 217 420-2

[39] Green N G, Ramos A, Gonzalez A, Morgan H and Castellanos A 2000 Phys. Rev. E 61 at press

[40] Gonzalez A, Ramos A, Green N G, Castellanos A and Morgan H 2000 Phys. Rev. E 61 at press

[41] Hiemenz P C 1986 Principles of Colloid and Surface Chemistry (New York: Dekker)

[42] Green N G, Ramos A, Morgan H and Castellanos A 1999 Electrostatics 99 (Cambridge, UK) (Inst. Phys. Conf. Ser.) 163 89-92

[43] Lyklema J 1991 Fundamentals of Interface and Colloid Science (London: Academic)

[44] Schwan H P 1992 Ann. Biomed. Eng. 20 269-88

[45] Zhou X F, Markx G H, Pethig R and Eastwood I M 1995 Biochim. Biophys. Acta 1245 85-93

[46] Stratton J A 1941 Electromagnetic Theory (New York: McGraw Hill)

[47] Lorrain P, Corson D R and Lorrain F 1988 Electromagnetic Fields and Waves (New York: Freeman)

[48] Wang X B, Huang Y, Becker F F and Gascoyne P R C 1994 J. Phys. D: Appl. Phys. 27 1571-4

[49] Melcher J R and Firebaugh M S 1967 Phys. Fluids 10 1178-85

[50] Ramos A, Gonzalez A, Castellanos A, Morgan H and Green N G 1999 Electrostatics 99 (Cambridge, UK) (Inst. Phys. Conf. Ser.) $163137-40$

[51] Huang Y and Pethig R 1991 Meas. Sci. Technol. 2 1142-6

[52] Green N G 1998 Dielectrophoresis of sub-micrometre particles $P h D$ Thesis University of Glasgow

[53] Schnelle T, Hagedorn R, Fuhr G, Fiedler S and Müller T 1993 Biochim. Biophys. Acta 1157 127-40

[54] Green N G, Ramos A, Gonzalez A, Castellanos A and Morgan H 2000 J. Phys. D: Phys. Appl. 33 L13-7 